\title{
Clinical Usefulness of Hybrid Intervention for the Treatment of Primary Thrombosis of Axillary-subclavian Vein
}

\author{
Shiro Onozawa, MD, Ph.D, Hiroyuki Tajima, MD, Ph.D, Satoru Murata, MD, Ph.D, \\ Ken Nakazawa, MD, Ph.D, and Tsuyoshi Fukunaga, MD
}

\begin{abstract}
Untreated symptomatic patients with Paget-Schroetter syndrome can suffer chronic disability due to venous obstruction, with arm swelling, pain, and early exercise fatigue. Although systemic or catheter-directed thrombolysis followed by anticoagulation and surgical intervention is recommended, there is no definite consensus about treatment. Here, we report the clinical usefulness of hybrid intervention with a combination of thrombectomy, thrombo-aspiration and balloon PTA, which has not been reported previously for this condition. These procedures were successful, and the patient has been free of symptoms for 6 years.
\end{abstract}

Keywords: interventional procedures, catheter and catheterization, Paget-Schroetter syndrome, thrombectomy

\section{INTRODUCTION}

$\mathrm{E}^{\mathrm{t}}$ ffort thrombosis or Paget-Schroetter syndrome most often affects young, active adults who are engaged in sports activities or whose professions require repetitive arm movements causing trauma to the axillary-subclavian vein and precipitating deep vein thrombosis. ${ }^{1,2)}$ Untreated symptomatic patients can suffer long-term disability due to venous obstruction, resulting in significant loss of occupational productivity and quality of life. ${ }^{3)}$ Systemic or catheter-directed thrombolysis followed by anticoagulation and surgical intervention has been recommended in much of the recent literature, there have been no reports of hybrid interventional treatment. ${ }^{4)}$ Here, we report the clinical usefulness of hybrid intervention involving a combination of percutaneous thrombectomy, thrombo-aspiration and balloon PTA for the treatment of Paget-Schroetter syndrome.

Departments of Radiology, Nippon Medical School, Tokyo, Japan

Received: March 21, 2008 Accepted: December 15, 2008.

Address for reprint requests to Shiro Onozawa, MD, Ph.D: Department of Radiology, Nippon Medical School, 1-1-5 Sendagi, Bunkyo-ku, Tokyo 113-8602, Japan

Tel: +81-35814-6240, Fax: +81-35685-1795

E-mail: onozawa@nms.ac.jp

\section{CASE}

A 61-year-old man presented at our hospital with a five-week history of untreated severe swelling and pain in the left upper extremity. He had undergone distal gastrectomy for gastric cancer 3 years previously, and had no coagulation disorder. Contrast venography on admission showed a massive filling defect of contrast medium in the left subclavian and axillary veins and collateral vessels (Fig. 1). Left upper extremity sub-acute thrombosis was diagnosed, and treatment was planned.

First, to avoid pulmonary thromboembolism, we introduced a temporary filter "Antheor" (Boston Scientific Japan, Tokyo, Japan) into the superior vena cava. We started systemic thrombolysis and anticoagulant therapy with urokinase 120,000 units/day and heparin sodium 12,000 units/day from the left forearm, compressing the swelling with elastic bandages.

Despite 5 days of treatment, the swelling of the upper extremity did not improve, and therefore we decided to add interventional procedures. First, a 5 Fr headhunter catheter (Medikit Co.Ltd, Tokyo, Japan) was inserted via the right femoral vein, and retrograde left subclavian venography was performed to evaluate the distribution and volume of the thrombi. Under guidance with a guidewire, we introduced an 8 Fr long sheath (Medikit Co.Ltd, Tokyo, Japan). A pigtail catheter was inserted, 


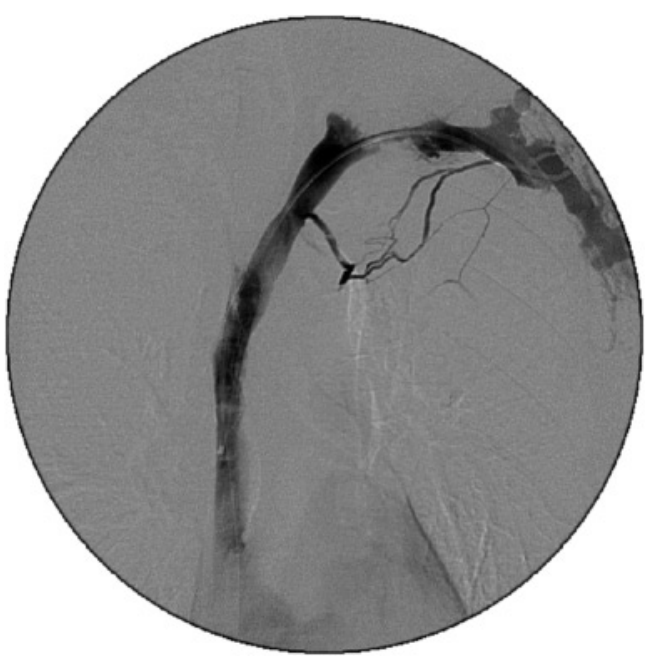

Fig. 1 Contrast venogram before interventions shows severe stenosis of the left subclavian vein.

and advanced or withdrawn by manual rotation (Fig. 2A). Thrombi were fragmented by the mechanical action of the rotating pigtail catheter. Manual clot aspiration was then performed using an 8Fr large-lumen percutaneous transluminal coronary angioplasty (PTCA) guide catheter "BriteTip" (Boston Scientific Japan, Tokyo, Japan). Strong manual aspiration was achieved via a regular Luer-Lok 20-mL syringe plunger (Becton Dickinson, Tokyo, Japan) while the catheter was slowly withdrawn through the introducer sheath. Finally, we added percutaneous transluminal angioplasty ( $8 \mathrm{~atm}, 30 \mathrm{~s}$, twice) using a balloon catheter $10 \mathrm{~mm}$ in diameter and $40 \mathrm{~mm}$ in length "Ultra-thin Diamond" (Boston Scientific Japan, Tokyo, Japan)(Fig. 2B). During these procedures, the patient received heparin sodium (initial dose, 5,000 IU; maintenance dose, activated partial thromboplastin time ratio of 2).

After disappearance of the massive thrombus had been confirmed (Fig. 2C), the temporary filter and sheath were withdrawn. Additional systemic heparin sodium infusion was administered for only seven days, and then oral warfarin sodium was started and continued for 8 months.

The patient's symptom improved, and he was discharged 27 days later. Histo-pathological examination showed that the aspirated thrombi were acute and organized. The patient is still attending as an outpatient, and no recurrence has been observed for 6 years.
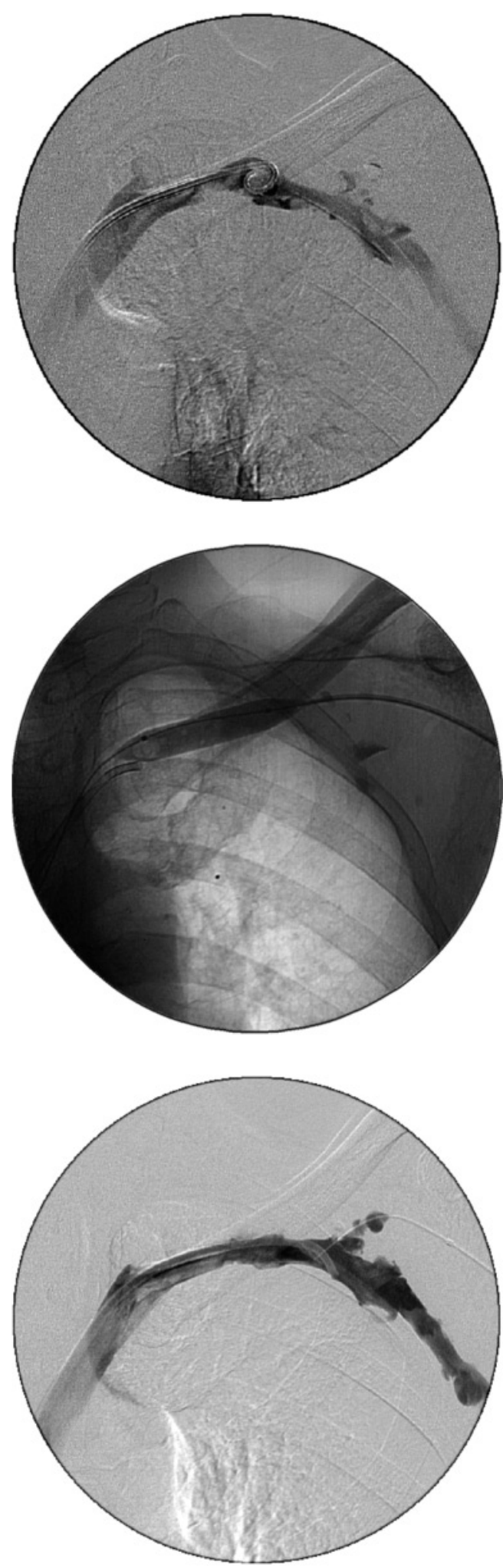

Fig. 2 Interventional procedures.

A: Manual thrombectomy with a rotating pigtail catheter.

B: Balloon angioplasty.

C: Post interventional venogram shows no stenosis of the left subclavian vein. 


\section{Discussion}

Although subclavian vein thrombosis is rather rare, it is a potentially dangerous acute vascular event representing 2-3\% of cases of deep venous thrombosis. The underlying causes differ widely, it may occur in patients with long-term implantation of venous catheters or pacemakers, as effort thrombosis in patients with thoracic outlet syndrome, or present in patients with thrombophilic disorders, but in most cases the causes are unknown. In the present case, the patient had slept with his arm elevated all night long after his gastrectomy because he believed in a therapeutic cult. This rather unusual situation may have created the venous compression, which was revealed by venography in a stress position (Fig. 3). Therefore we decided to stop his strange custom.

There is no consensus regarding the optium treatment for Paget-Schroetter syndrome because of difficulty in obtaining an adequate number of patients for an acceptable prospective randomized trial. A multidisciplinary strategy, involving a vascular surgeon, interventional radiologist and haematologist should be adopted for management. $^{4)}$

As to the indication of interventional treatment for this disease is not also confirmed, but at least unimproved symptoms in spite of systemic thrombolysis must be one of the indications of intervention. Our patient had a 5-week history of untreated thrombosis, and systemic thrombolysis for 5 days was unable to clear the mature massive thrombus. Because of the presence of collateral pathways, it was difficult for the thrombolytic drugs to reach the thrombus, and the elastic bandage would not have helped to reduce the collaterals for anatomical reasons.

Mechanical thrombectomy is a novel approach for the treatment of venous thrombosis. Several small studies have shown that mechanical thrombectomy devices can be used to remove venous thrombi quickly and effectively in the treatment of deep venous thrombosis, pulmonary thromboembolism, and internal jugular vein thrombosis. ${ }^{6)}$ The technique of fragmentation with a special pigtail catheter system has already been described. The rotational movement of the pigtail portion of the catheter acts directly on the clots in the vein, causing fragmentation and migration of the smaller fragments. ${ }^{7)}$ Mechanical thrombectomy combined with thrombolysis has shown good results. ${ }^{8)}$

Percutaneous aspiration evolved from a simple technique used previously in many fields. ${ }^{9)}$ The thin wall of

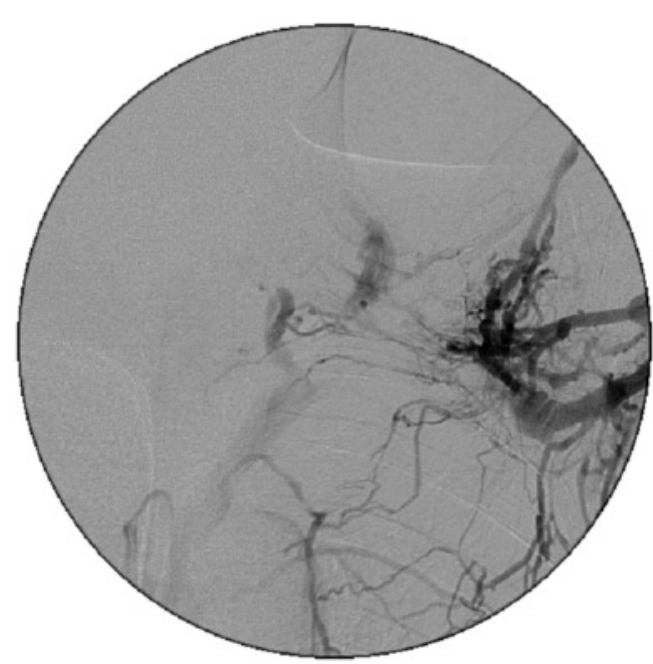

Fig. 3 Contrast venogram with stress position on admission shows complete occlusion of the left subclavian vein and collateral formaions.

the aspiration catheter ensures that the internal luminal diameter is maximal for aspiration of the thrombus. This technique is less invasive for vessels and is convenient to perform in a standard angiography laboratory with the use of a small $8 \mathrm{Fr}$ introducer sheath and a conventional PTCA guide catheter. It is also inexpensive.

Thrombectomy and thrombo-aspiration may have a possible synergistic effect with concurrent thrombolytic therapy because a large surface area of the resulting clot fragments is exposed to the thrombolytic agent, thus improving the results of lytic activity and allowing reduction of the dose and infusion time. ${ }^{6}$ In our patient, use of thrombolytic therapy was unnecessary because of the excellent results of thrombectomy and thrombo-aspiration.

After complete clot removal, it is necessary to perform a final dilatation of the stenotic orifice of the subclavian vein. The main purpose of this procedure is to maintain an efficient central channel in the orifice of the vessel. Because in the present case the dilatation was effective, we did not have to use metallic stents. For Paget-Schroetter syndrome, stents seem to be of little value and are associated with a high incidence of stent fractures. ${ }^{10)}$

In conclusion, the present case shows that percutaneous interventional procedures can achieve a rapid and safe improvement in the venous circulation of patients with Paget-Schroetter syndrome. 


\section{REFERENCES}

1) Youssef MY, Al Taweel T, Asfar S, Abdella N. Effortinduced venous thrombosis of the upper limbs. Med Princ Pract. 2006; 15: 299-302.

2) Urschel HC Jr, Razzuk MA. Improved management of the Paget-Schroetter syndrome secondary to thoracic outlet compression. Ann Thorac Surg. 1991; 52: $1217-21$.

3) Khan SN, Stansby G. Current management of PagetSchroetter syndrome in the UK. Ann R Coll Surg Engl. 2004; 86: 29-34.

4) Sajid MS, Ahmed N, Desai M, Baker D, Hamilton G. Upper limb deep vein thrombosis: a literature review to streamline the protocol for management. Acta Haematol. 2007; 118: 10-8.

5) Sabeti S, Schillinger M, Mlekusch W, Haumer M, Ahmadi R, Minar E. Treatment of subclavian-axillary vein thrombosis: long-term outcome of anticoagulation versus systemic thrombolysis. Thromb Res. 2002; 108: $279-85$.

6) Tajima H, Murata S, Kumazaki T, Ichikawa K, Tajiri T, Yamamoto Y. Successful interventional treatment of acute internal jugular vein thrombosis. AJR Am J Roentgenol. 2004; 182: 467-9.

7) Schmitz-Rode T, Janssens U, Duda SH, Erley CM, Günther RW. Massive pulmonary embolism: percutaneous emergency treatment by pigtail rotation catheter. J Am Coll Cardiol. 2000; 36: 375-80.

8) Gaffar M. Upper extremity deep vein thrombosis. Hosp Physician. 2005; 6: 29-34.

9) Morgan R, Belli AM. Percutaneous thrombectomy: a review. Eur Radiol. 2002; 12: 205-17.

10) Urschel HC Jr, Razzuk MA. Improved management of the Paget-Schroetter syndrome secondary to thoracic outlet compression. Ann Thorac Surg. 1991; 52: $1217-21$. 